\title{
Cochrane Review Summary: Family-based programmes for preventing smoking by children and adolescents
}

\author{
NCF \\ COCHRANE \\ Nursing Care \\ Field
}

\section{Daksha Trivedi}

Senior Research Fellow, Centre for Research in Primary and Community Care, University of Hertfordshire, Hatfield, UK

Key words: adolescent; children; prevention; programme; smoking

First published online 15 March 2017

\section{Review question}

Are family-based programmes effective for preventing smoking in children and adolescents?

\section{Relevance to primary care and nursing}

Primary health-care professionals including nurses have an active role to promote healthy behaviour among children and young people. This includes preventing uptake of smoking and working with families, schools and the wider community sectors, promoting awareness and giving advice about tobacco-free lifestyles (National Institute for Health and Clinical Excellence, 2015).

\section{Characteristics of the evidence}

This Cochrane review contained 27 randomised controlled trials (RCTs) of which 12 were randomised and 15 were cluster RCTs (Thomas et al., 2015). In total, 23 were based in the United States, two in Europe and two in Australia and India, respectively.

Included studies targeted children (aged 5-12) or adolescents (aged 13-18), and family members who were offered interventions to prevent initiation of smoking. School- or community-based interventions, or those that also examined the prevention of drug or alcohol use were included provided they had a

Correspondence to: Dr Daksha Trivedi, Senior Research Fellow, Centre for Research in Primary and Community Care, University of Hertfordshire, College Lane, Hatfield AL10 9AB, UK. Email: d.trivedi@herts.ac.uk

(C) Cambridge University Press 2017 clear family-based component and measured outcomes for tobacco use with at least six months' follow-up from the start of intervention. Excluded studies were those that did not provide baseline smoking status, smoking behaviour at follow-up, did not focus on prevention or did not enable the analysis to separate family intervention effects from other components.

Family interventions were heterogeneous, varied in intensity and mostly addressed family functioning for the prevention of risky behaviours which included tobacco use. Interventions were compared with no intervention or usual care, or as an adjunct to a school-based intervention.

\section{Summary of key evidence}

The evidence was judged to be of moderate quality with an overall uncertainty in the results, using GRADE (Grading of Recommendations Assessment, Development and Evaluation) criteria. Meta-analysis was conducted where appropriate. The primary outcome was smoking behaviour. Duration of follow-up post intervention ranged from six months to more than 15 years. Sample size $(n)$, risk ratios (RR) and $95 \%$ confidence intervals (CI) are given where appropriate.

Programme intensity was rated as high, medium or low according to the level of proximity (high if delivered on site or face to face; low if mailing or telephone); direction (high if directed with contacts, low if self-directed); exposure period (high if duration of intervention and number of components were high); unit of delivery (high 
if family groups or individual families, low if community level). Low-intensity programmes were usually written materials or brief contact. No studies reported any adverse events from the interventions.

\section{Family-based interventions compared with no intervention}

Evidence from nine studies showed significantly reduced uptake of smoking for baseline never smokers who received family-based interventions ( $n=4810$ at follow-up, RR 0.76 , 95\% CI $0.68-$ $0.84)$. Of these, six high-intensity interventions reported a significant effect (RR $0.71,95 \%$ CI $0.61-0.82$ ). One medium-intensity intervention and two low-intensity interventions showed similar effects favouring the intervention, but with wider CI. The estimated benefit of standalone interventions was reported as a reduction in new smoking behaviour of $16-32 \%$.

\section{Combined family plus school intervention compared with school intervention}

There was evidence of benefit from two studies for the combined intervention $(n=2301$ at followup, RR 0.85, 95\% CI 0.75-0.96). One highintensity intervention study reported a significant effect of the additional intervention, although some participants reported being smokers at baseline ( $n=1096$, RR $0.60,95 \%$ CI $0.38-0.94)$. Five other studies, not pooled in a meta-analysis ( $n=\sim 18500$ comprising high-, medium- and lowintensity studies), showed no significant effects. The estimated benefit of combined interventions was reported as a reduction in new smoking behaviour of $4-25 \%$.

Key characteristics of effective high-intensity interventions include authoritative parenting (caring and supervised parenting), frequent contacts with general practitioners to enhance positive qualities in new mothers, motivating parents and families to engage in services, telephone facilitators supporting parents and their children working together, positive parenting and family support and strengthening their skills. Effective combined interventions also included strategies for effective communication and freedom to choose and make one's own decisions.

Primary Health Care Research \& Development 2017; 18: 303-304

\section{Implications for practice}

Family-based high-intensity interventions can be effective in preventing children and adolescents from starting to smoke. Authoritative parenting interventions can be delivered to families who engage well, if resources are available. However, implementation integrity and outcomes need to be monitored given the heterogeneity of interventions and settings.

\section{Implications for research}

Further research should examine components of the theoretically based interventions and evaluate them in adequately powered trials with different adolescent and family population groups in local settings and contexts. Minimising attrition, maximising intervention fidelity and assessing clustering effects are important. High-quality economic evaluations are required to assess cost-effectiveness of these interventions.

\section{Acknowledgements}

The author is a member of the Cochrane Nursing Care Field (CNCF).

\section{Financial Support}

This research received no specific grant from any funding agency, commercial or not-for-profit sectors.

\section{Conflicts of Interest}

None.

\section{References}

National Institute for Health and Clinical Excellence 2015: Smoking: preventing uptake in children and young people. Public Health Guideline [PH14], updated November 2014. Retrieved 9 December 2016 from https://www.nice.org.uk/ guidance/ph14.

Thomas, R.E., Baker, P.R.A., Thomas, B.C. and Lorenzetti, D.L. 2015: Family-based programmes for preventing smoking by children and adolescents. Cochrane Database of Systematic Reviews, Issue 2, Art. No. CD004493. https://doi.org/10.1002/ 14651858.CD004493.pub3. 